\title{
The critical values of certain zeta functions associated with modular forms of half-integral weight
}

\author{
By Goro SHIMURA*
}

(Received March 24, 1980)

\section{Introduction.}

The main theme of this paper is the algebraicity of two types of numbers occurring in connection with modular forms. One is the values at certain integers or half-integers of a Dirichlet series

$$
D(s, f, g)=\sum_{n=1}^{\infty} a(n) b(n) n^{-s}
$$

obtained from two modular forms

$$
f(z)=\sum_{n=1}^{\infty} a(n) e^{2 \pi i n z} \quad \text { and } \quad g(z)=\sum_{n=0}^{\infty} b(n) e^{2 \pi i n z},
$$

and the other is the inner product $\langle f, g\rangle$ of $f$ and $g$, when they have the same weight. These have been treated in our previous papers [12], [13], and [14] for the forms $f$ and $g$ of integral weight. Therefore the present investigation concerns the cases in which either or both of $f$ and $g$ have half-integral weight.

To describe the nature of the problems as well as of the results, we let $m^{\prime}$ and $m$ denote the weights of $f$ and $g$, respectively, which are positive elements of $2^{-1} \boldsymbol{Z}$. If $m=m^{\prime}$, there is a well known relation between $\langle f, g\rangle$ and the residue of $D(s, f, g)$ at $s=m$, and therefore the second object has a character similar to the first one. Setting this residue aside, we restrict, for some natural reasons, the study of the values of $D(s, f, g)$ to the case $m<m^{\prime}$. If we exclude the case in which both $m$ and $m^{\prime}$ are integers, there are three cases:

I . $m \notin \boldsymbol{Z}$ and $m^{\prime} \in \boldsymbol{Z}$,

II. $m \in \boldsymbol{Z}$ and $m^{\prime} \notin \boldsymbol{Z}$,

III. $m \in \boldsymbol{Z}$ and $m^{\prime} \in \boldsymbol{Z}$.

The first case was treated by Sturm [17], [18]. Therefore we consider here the remaining two cases. Let $m^{\prime}=k / 2$ with an odd integer $k$, and assume that $f$

\footnotetext{
* Supported by NSF Grant MCS 7903631.
} 
is a cusp form which is an eigenfunction of the operators $T\left(p^{2}\right)$ introduced in [9] for almost all primes $p$; put $f \mid T\left(p^{2}\right)=c_{p} f$. The main theorem of [9] shows that there is a modular form $F$ of integral weight $k-1$ such that $F \mid T(p)=c_{p} F$ for all such $p$, where $T(p)$ is the ordinary Hecke operator of degree $p$ and of weight $k-1$. To obtain the algebraicity, we naturally assume that $a(n)$ and $b(n)$ are all algebraic numbers. We shall then show that both $\langle f, g\rangle$ (when $m=m^{\prime}=k / 2$ ) and the values of $D\left(s / 2, f, g\right.$ ) (when $m<m^{\prime}$ ) for certain integers $s$ are algebraic numbers times $\pi^{n / 2} u_{-}(F)$, where $n$ is an integer depending on $s, m, m^{\prime}$, and $u_{-}(F)$ is a "period" of $F$. Actually we prove a stronger result which tells the behavior of the algebraic numbers under automorphisms.

The methods of proof are, for the most part, the same as in our previous papers [12], [13], [14] and also in Sturm [17]. However, we are confronted by a new difficulty, since no satisfactory theory of "primitive forms" has been developed in the case of half-integral weight. Such a theory in the case of integral weight was needed in order to obtain the most general result without any artificial conditions. Therefore one of the main technical objectives of this work is to show that the concept of "primitive form" is unnecessary, at least for our present purposes. In fact, we shall obtain our results for an arbitrary eigenfunction $f$ of $T\left(p^{2}\right)$ for almost all $p$. (We can assume, however, that $F$ is primitive.) The dispensability of the concept of primitive form is significant, because it will make the study of the critical values of the zeta functions associated with modular forms of other types practicable. In fact, our technique in the proof of Theorem 1 seems applicable, for example, to the Siegel modular forms. As another technical necessity dictated by our methods, we have to analyse carefully the behavior of modular forms under the action of automorphisms of $\boldsymbol{C}$ on the Fourier coefficients.

Since both inner products and the values of zeta functions are natural objects of arithmetic interest, their investigation hardly needs justification. It may be added, however, that there is an interesting application of our results to the theory of algebraic curves uniformized by automorphic functions of arithmetic type, which the author hopes to treat in a subsequent paper. Though the lemmas are of course preliminaries to the main theorems, some of them (Lemmas 9 and 10, for example) may be of independent interest.

\section{Statement of the main results.}

We denote by $G L_{2}^{+}(\boldsymbol{R})$ the subgroup of $G L_{2}(\boldsymbol{R})$ consisting of the elements with positive determinant, and let every element $\alpha=\left(\begin{array}{ll}a & b \\ c & d\end{array}\right)$ of $G L_{2}^{+}(\boldsymbol{R})$ act on the upper half plane 


$$
H=\{z \in C \mid \operatorname{Im}(z)>0\}
$$

by the rule $\alpha(z)=(a z+b) /(c z+d)$. For every positive integer $N$, we define congruence subgroups $\Gamma_{0}(N), \Gamma_{1}(N)$, and $\Gamma(N)$ of $S L_{2}(\boldsymbol{Z})$ by

$$
\begin{aligned}
& \Gamma_{0}(N)=\left\{\left(\begin{array}{ll}
a & b \\
c & d
\end{array}\right) \in S L_{2}(\boldsymbol{Z}) \mid c \equiv 0(\bmod N)\right\}, \\
& \Gamma_{1}(N)=\left\{\left(\begin{array}{ll}
a & b \\
c & d
\end{array}\right) \in \Gamma_{0}(N) \mid a \equiv d \equiv 1(\bmod N)\right\}, \\
& \Gamma(N)=\left\{\left(\begin{array}{ll}
a & b \\
c & d
\end{array}\right) \in \Gamma_{1}(N) \mid b \equiv 0(\bmod N)\right\} .
\end{aligned}
$$

We introduce a factor of automorphy $j(\gamma, z)$ of weight $1 / 2$ for $\gamma \in \Gamma_{0}(4)$ by

$$
\begin{gathered}
\theta(\gamma(z))=j(\gamma, z) \theta(z) \quad\left(\gamma \in \Gamma_{0}(4)\right), \\
\theta(z)=\sum_{n=-\infty}^{\infty} e\left(n^{2} z\right) .
\end{gathered}
$$

Here and throughout the paper, we put

We recall that

$$
e(z)=\exp (2 \pi i z) \quad(z \in C) .
$$

$$
j\left(\left(\begin{array}{ll}
a & b \\
c & d
\end{array}\right), z\right)^{2}=\left(\frac{-1}{d}\right)(c z+d) \quad \text { (see [9]). }
$$

Given a non-negative element $\lambda$ of $2^{-1} \boldsymbol{Z}$, an element $\gamma=\left(\begin{array}{ll}* & * \\ c & d\end{array}\right)$ of $S L_{2}(\boldsymbol{Z})$, and a function $f$ on $H$, we define a function $\left.f\right|_{2} \gamma$ on $H$ by

$$
\left(\left.f\right|_{\lambda} \gamma\right)(z)=\left\{\begin{array}{lll}
f(\gamma z)(c z+d)^{-\lambda} & \text { if } \quad \lambda \in \boldsymbol{Z}, \\
f(\gamma z) j(\gamma, z)^{-2 \lambda} & \text { if } \lambda \notin \boldsymbol{Z} .
\end{array}\right.
$$

We naturally assume that $\gamma \in \Gamma_{0}(4)$ when $\lambda \notin Z$. For a congruence subgroup $\Gamma$ of $S L_{2}(\boldsymbol{Z})$, which we assume to be contained in $\Gamma_{0}(4)$ if $\lambda \notin \boldsymbol{Z}$, we denote by $\mathscr{M}(\lambda, \Gamma)$ the set of all modular forms of weight $\lambda$ with respect to $\Gamma$, that is, holomorphic functions $f$ on $H$ such that

$$
\left.f\right|_{\lambda} \gamma=f \text { for all } \gamma \in \Gamma,
$$

$f$ is holomorphic at every cusp.

We call such an $f$ a cusp form if $f$ vanishes at every cusp, and denote by $\mathcal{S}(\lambda, \Gamma)$ the set of all cusp forms of $\mathscr{M}(\lambda, \Gamma)$. The reader is referred to [9] for the precise meaning of these cusp conditions. We then put 


$$
\begin{array}{ll}
\mathscr{M}(\lambda, N)=\mathscr{M}\left(\lambda, \Gamma_{1}(N)\right), & \mathcal{S}(\lambda, N)=\mathcal{S}\left(\lambda, \Gamma_{1}(N)\right), \\
\mathscr{M}(\lambda)=\bigcup_{N=1}^{\infty} \mathscr{M}(\lambda, \Gamma(N)), & \mathcal{S}(\lambda)=\bigcup_{N=1}^{\infty} \mathcal{S}(\lambda, \Gamma(N))
\end{array}
$$

$(4 \mid N$ if $\lambda \notin \boldsymbol{Z})$. Given a character $\chi$ modulo $N$, we put

$$
\begin{aligned}
& \mathscr{M}(\lambda, N, \chi)=\left\{f \in \mathscr{M}(\lambda, N)\left|\chi\left(d_{\gamma}\right) f=f\right|_{\lambda} \gamma \text { for every } \gamma \in \Gamma_{0}(N)\right\}, \\
& \mathcal{S}(\lambda, N, \chi)=\mathcal{S}(\lambda) \cap \mathscr{M}(\lambda, N, \chi) .
\end{aligned}
$$

Here and throughout the paper, $d_{\gamma}$ denotes the lower right entry of $\gamma$. We always assume

$$
\begin{aligned}
& \chi(-1)=(-1)^{\lambda} \quad \text { if } \quad \lambda \in \boldsymbol{Z}, \\
& \chi(-1)=1 \quad \text { and } \quad 4 \mid N \text { if } \lambda \notin \boldsymbol{Z},
\end{aligned}
$$

because $\mathscr{M}(\lambda, N, \chi)=\{0\}$ otherwise.

Introducing an invariant measure $d \mu(z)$ on $H$ by

$$
d \mu(z)=y^{-2} d x d y, \quad z=x+i y,
$$

we define, for two elements $f$ and $g$ of $\mathscr{M}(\lambda)$, their inner product $\langle f, g\rangle$ by

$$
\begin{aligned}
& \langle f, g\rangle=\mu(\Phi)^{-1} \int_{\Phi} \overline{f(z)} g(z) y^{2} d \mu(z), \\
& \mu(\Phi)=\int_{\Phi} d \mu(z), \quad \Phi=\Gamma(N) \backslash H,
\end{aligned}
$$

where we take $N$ so that both $f$ and $g$ belong to $\mathscr{M}(\lambda, \Gamma(N))$. To guarantee the convergence of the integral, we assume that either $f$ or $g$ is a cusp form. We note that

$$
\mu(\Gamma(N) \backslash H)=(\pi / 3)[\Gamma(1): \Gamma(N)\{ \pm 1\}] .
$$

We also define the symbol $\langle f, g\rangle$ by (1.5) for continuous functions $f$ and $g$ such that $\left.f\right|_{\lambda} \gamma=f$ and $\left.g\right|_{\lambda} \gamma=g$ for all $\gamma \in \Gamma(N)$, whenever the integral is convergent.

Every element $f$ of $\mathscr{N}(\lambda, \Gamma(N))$ has a Fourier expansion

$$
f(z)=\sum_{n=0}^{\infty} a(n) e(n z / N) .
$$

Let $\operatorname{Aut}(\boldsymbol{C})$ denote the group of all ring-automorphisms of the complex number field $\boldsymbol{C}$, and let $\sigma \in \operatorname{Aut}(\boldsymbol{C})$. Then we can define an element $f^{\sigma}$ of $\mathscr{M}(\lambda)$ by

$$
f^{\sigma}(z)=\sum_{n=0}^{\infty} a(n)^{\sigma} e(n z / N) .
$$

Such an action can be defined even for Siegel modular forms of integral and 
half-integral weights (see [15]). If $f$ is a cusp form, so is $f^{\sigma}$. This is proved in [14, Proposition 1.8] when $\lambda \in Z$. The case of half-integral weight can be reduced to that of integral weight by taking the square of a given form. In particular, we denote by $\rho$ the complex conjugation, and we put thus

$$
f^{\rho}(z)=\sum_{n=0}^{\infty} \overline{a(n)} e(n z / N)=\overline{f(-\bar{z})} .
$$

We see easily that

$$
\left\langle f^{\rho}, g\right\rangle=\left\langle g^{\rho}, f\right\rangle .
$$

We say that $f$ is rational over a subfield $L$ of $\boldsymbol{C}$, or $f$ is $L$-rational, if $L$ contains all the coefficients $a(n)$. This is so if and only if $f^{\sigma}=f$ for all $\sigma$ of $\operatorname{Aut}(\boldsymbol{C})$ which give the identity map on $L$. Given modular forms $f, g, \cdots$ and Dirichlet characters $\chi, \phi, \cdots$, we denote by $K(f, g, \cdots, \chi, \phi, \cdots)$ the field generated over $\boldsymbol{Q}$ by the Fourier coefficients of $f, g, \cdots($ at $i \infty)$ and the values of $\chi, \phi, \cdots$.

Suppose $\lambda \in Z$; let

$$
F(z)=\sum_{n=1}^{\infty} c(n) e(n z) \in \bigcup_{N=1}^{\infty} \mathcal{S}(\lambda, N)
$$

and let $\psi$ be a primitive character modulo a positive integer $r$. Then we put

$$
\begin{aligned}
& D(s, F)=\sum_{n=1}^{\infty} c(n) n^{-s}, \\
& D(s, F, \phi)=\sum_{n=1}^{\infty} \psi(n) c(n) n^{-s} .
\end{aligned}
$$

We call $F$ primitive if the following conditions are satisfied:

(1.10a) $\quad F$ is a common eigenfunction of Hecke operators $T(p)$ for almost all primes $p$ (that is, for all except a finite number of $p$ );

$(1.10 \mathrm{~b}) \quad c(1)=1$;

(1.10c) There is a positive integer $N$ such that $F \in \mathcal{S}(\lambda, N)$ and that $\langle F, h(t z)\rangle=0 \quad$ for all $h \in \mathcal{S}(\lambda, M), t M \mid N, M<N$.

The reader is referred to Miyake [3] for the basic results on primitive forms. We recall in particular that such a form $F$ is an eigenfunction of Hecke operators $T(p)$ of level $N$ for all primes $p ; F \mid T(p)=c(p) F ; F$ is uniquely determined by giving the eigenvalues $c(p)$ for almost all $p$. Moreover, if $F$ is a primitive element belonging to $\mathcal{S}(\lambda, N, \chi)$, then $F^{\sigma}$ is primitive and belongs to $\mathcal{S}\left(\lambda, N, \chi^{\sigma}\right)$ (see [14, Proposition 2.6]).

Now let $k$ be an odd positive integer. In [9], we defined a certain operator 
$T_{k, \chi}^{N}\left(p^{2}\right)$ on $\mathscr{M}(k / 2, N, \chi)$, which we simply write $T_{N}\left(p^{2}\right)$ or $T\left(p^{2}\right)$ when there is no fear of confusion. Suppose $k \geqq 3$ and an element $g$ of $\mathcal{S}(k / 2, N, \chi)$ is an eigenfunction of $T_{N}\left(p^{2}\right)$ for all primes $p$, and let $g \mid T\left(p^{2}\right)=b(p) g$. Then the main theorem of [9] shows that there is an element $h$ of $\mathscr{M}\left(k-1, N^{\prime}, \chi^{2}\right)$ with some $N^{\prime}$ such that

$$
D(s, h)=\prod_{p}\left[1-b(p) p^{-s}+\chi(p)^{2} p^{k-2-2 s}\right]^{-1} .
$$

Moreover, $h$ is a cusp form if $k \geqq 5$. With this result in mind, we introduce a new concept as follows.

Suppose $k \geqq 3$. Fix a primitive element $F$ of $S(k-1)$ with eigenvalues $c(p)$ as above, or rather, fix a system of eigenvalues $\{c(p)\}$. We say that an element $f$ of $\mathcal{S}(k / 2, N, \chi)$ corresponds to $F$, or to $\{c(p)\}$, if $f \mid T\left(p^{2}\right)=c(p) f$ for almost all $p$. Then we denote by $\mathcal{S}(k / 2, N, \chi, F)$ the vector subspace of $\mathcal{S}(k / 2, N, \chi)$ consisting of all the elements corresponding to $F$. In $\S 2$, we shall prove that this subspace is spanned by $K(\chi, F)$-rational elements, and sent to $\mathcal{S}\left(k / 2, N, \chi^{\sigma}, F^{\sigma}\right)$ by $\sigma$.

Given an arbitrary primitive element $h$ of $\mathcal{S}(\lambda)$ and a primitive character $\phi$, we treated in our previous papers the values of $D(s, h, \phi)$ for certain integers $s$. To recall the result, put

$$
A(m, h, \phi)=(2 \pi i)^{-m} \tau(\psi)^{-1} D(m, h, \psi)
$$

for every integer $m$ in the range $0<m<\lambda$, where $\tau(\psi)$ is the Gauss sum defined as usual by

$$
\tau(\psi)=\sum_{n=1}^{r} \psi(n) e(n / r) \quad(r=\text { conductor of } \psi) .
$$

If $\psi^{\prime}$ is an imprimitive character associated with $\phi$, we define its Gauss sum $\tau\left(\psi^{\prime}\right)$ to be the same as $\tau(\psi)$. Now notice that the number of all different $h^{\sigma}$ with $\sigma \in \operatorname{Aut}(\boldsymbol{C})$ is $[K(h): \boldsymbol{Q}]$. We proved in [13] (see also [12], [14]) that there exist $2[K(h): Q]$ complex numbers $u_{ \pm}\left(h^{\sigma}\right)$ depending only on $h^{\sigma}$ with the following properties:

$$
\begin{aligned}
& A(m, h, \phi) / u_{+}(h) \in K(h, \phi) \text { if } \phi(-1)=(-1)^{m}, \\
& A(m, h, \phi) / u_{-}(h) \in K(h, \psi) \text { if } \phi(-1)=(-1)^{m-1} \\
& \text { for every positive integer } m<\lambda .
\end{aligned}
$$

$$
\left[A(m, h, \phi) / u_{ \pm}(h)\right]^{\sigma}=A\left(m, h^{\sigma}, \phi^{\sigma}\right) / u_{ \pm}\left(h^{\sigma}\right) \text { for every } \sigma \in \operatorname{Aut}(\boldsymbol{C}),
$$
where the choice between $u_{+}$and $u_{-}$is given as in (1.12a).

After these preliminaries, our first main result can be stated as follows. 
THEOREM 1. Suppose $k$ is an odd integer $\geqq 3$. For $f \in \mathcal{S}(k / 2, N, \chi, F)$ and $g \in \mathcal{S}(k / 2, N)$, put

$$
I(f, g)=\pi i \tau(\chi)\langle f, g\rangle .
$$

Then $I(f, g) / u_{-}(F) \in K\left(f^{o}, g\right)$. Moreover, for every $\sigma \in \operatorname{Aut}(\boldsymbol{C})$, we have

$$
\left[I(f, g) / u_{-}(F)\right]^{\sigma}=I\left(f^{\rho \sigma \rho}, g^{\sigma}\right) / u_{-}\left(F^{\sigma}\right) .
$$

In particular, if $f$ is $K(F, \chi)$-rational, then $I(f, g) / u_{-}(F)$ belongs to $K(F, \chi, g)$, and we have, for every $\sigma \in \operatorname{Aut}(\boldsymbol{C})$,

$$
\left[I(f, g) / u_{-}(F)\right]^{\sigma}=I\left(f^{\sigma}, g^{\sigma}\right) / u_{-}\left(F^{\sigma}\right) .
$$

This will be proved in $\S 4$.

To state the second main result, we define, for two modular forms

$$
\begin{aligned}
& f(z)=\sum_{n=1}^{\infty} a(n) e(n z) \in \mathcal{S}\left(m^{\prime}, N, \chi\right), \\
& g(z)=\sum_{n=0}^{\infty} b(n) e(n z) \in \mathcal{M}(m, N, \phi),
\end{aligned}
$$

a Dirichlet series $D(s, f, g)$ by

$$
D(s, f, g)=\sum_{n=1}^{\infty} a(n) b(n) n^{-s} .
$$

This is convergent for sufficiently large $\operatorname{Re}(s)$, and can be continued to a meromorphic function on the whole plane. In [12] and [13], we examined the critical values of $D(s, f, g)$ when both $m$ and $m^{\prime}$ are integers and $m<m^{\prime}$. Sturm treated the case where $m<m^{\prime} \in \boldsymbol{Z}, m \in \boldsymbol{Z}$ (see [18]). We consider here the following cases:

(I) $m^{\prime}=k / 2$ and $m=l / 2$ with odd integers $k$ and $l ; k>l, k \geqq 5$.

(II) $m^{\prime}=k / 2, m \in \boldsymbol{Z}$, and $m \leqq(k-3) / 2$.

Let us now put, in Case ( $\mathrm{I}$ ),

$$
\mathscr{D}_{N}(s, f, g)=L_{N}(2 s+2-(k+l) / 2, \omega) D(s, f, g),
$$

where $\omega(d)=\left(\frac{-1}{d}\right)^{\lambda} \chi(d) \psi(d), \lambda=(k-l) / 2$, and

$$
L_{N}(s, \omega)=\sum_{n>0,(n, N)=1} \omega(n) n^{-s} ;
$$

we put subscript $N$ to emphasize the condition $(n, N)=1$. This notation $L_{N}$ applies to an arbitrary primitive or imprimitive character $\omega$ whose conductor may or may not divide $N$. 
TheOREM 2. Under assumption ( I ), $\Gamma(s) \Gamma(s+1-(l / 2)) \mathscr{D}_{N}(s, f, g)$ is an entire function. Suppose moreover that $f \in \mathcal{S}(k / 2, N, \chi, F)$; let $n$ be an odd integer such that

$$
l \leqq n \leqq k-2,
$$

and let

$$
B(n, f, g)=i \tau(\phi)^{-1} \pi^{(l-1-2 n) / 2} \mathscr{D}_{N}(n / 2, f, g) / u_{-}(F) .
$$

Then $B(n, f, g) \in K(f, g)$. Moreover, for every $\sigma \in \operatorname{Aut}(\boldsymbol{C})$, we have

$$
B(n, f, g)^{\sigma}=B\left(n, f^{\sigma}, g^{\sigma}\right) \text {. }
$$

The existence of the factor $\Gamma(s+1-(l / 2))$ shows that $\mathscr{D}_{N}(n / 2, f, g)=0$ for every odd integer $n$ less than $l$.

THEOREM 3. Let $f \in \mathcal{S}(k / 2, N, \chi, F)$ and $g \in \mathscr{M}(m, N, \phi)$ with an odd integer $k$ and an integer $m<k / 2$. Put $\mu=(k+2 m-1) / 4$ and

Then

$$
\mathscr{D}_{N}(s, f, g)=L_{N}\left(4 s-4 \mu+2, \chi^{2} \psi^{2}\right) D(s, f, g) .
$$

$$
(s-\mu) \Gamma(s) \Gamma(s+1-m) \Gamma(s-\mu+(1+\varepsilon) / 2) \mathscr{D}_{N}(s, f, g)
$$

is an entire function, where $\varepsilon$ is 0 or 1 according as $2 \mu$ is even or odd. The factor $s-\mu$ is unnecessary if $2 \mu$ is odd or if $\chi^{2} \psi^{2}$ is nontrivial. Moreover, put

$$
\begin{aligned}
& J_{1}=\left\{\begin{array}{l}
\{n \in Z \mid m+(k-1) / 2 \leqq n \leqq k-2, n \leqq 1(\bmod 2)\} \text { if } \chi^{2} \psi^{2} \text { is nontrivial } \\
\{n \in Z \mid m+(k-1) / 2<n \leqq k-2, n \equiv 1(\bmod 2)\} \text { if } \chi^{2} \psi^{2} \text { is trivial, }
\end{array}\right. \\
& J_{2}=\{n \in Z \mid 2 m \leqq n \leqq m+(k-3) / 2, n \equiv 0(\bmod 2)\}, \\
& C(n, f, g)=\left\{\begin{array}{l}
i \tau\left(\chi \psi^{2}\right)^{-1} \pi^{2 m-2 n+(k-3) / 2} \mathscr{D}_{N}(n / 2, f, g) / u_{-}(F) \text { for } n \in J_{1}, \\
i^{m-1} \tau(\psi)^{-1} \pi^{m-n-1} \mathscr{D}_{N}(n / 2, f, g) / u_{-}(F) \text { for } n \in J_{2} .
\end{array}\right.
\end{aligned}
$$

Then $C(n, f, g)^{\sigma}=C\left(n, f^{\sigma}, g^{\sigma}\right)$ for every $\sigma \in \operatorname{Aut}(\boldsymbol{C})$.

We see from the nature of the gamma factors that

$$
\begin{aligned}
& \mathscr{D}_{N}(n / 2, f, g)=0 \text { if } n \equiv 1(\bmod 2) \text { and } n \leqq m+(k-3) / 2, \\
& \text { or if } n \equiv 0(\bmod 2) \text { and } n \leqq 2 m-2 .
\end{aligned}
$$

Obviously $J_{1} \cup J_{2}$ is empty unless $k-2 m \geqq 3$. These two theorems will be proved in $\S 5$.

\section{The behavior of modular forms under $\operatorname{Aut}(C)$.}

Throughout the rest of the paper, we denote by $\overline{\boldsymbol{Q}}$ the algebraic closure of $\boldsymbol{Q}$ and by $\boldsymbol{Q}_{\mathrm{ab}}$ the maximal abelian extension of $\boldsymbol{Q}$, both embedded in $\boldsymbol{C} ; \boldsymbol{A}$ will 
denote the adeles of $\boldsymbol{Q}$.

We introduce a group $\mathcal{G}$ consisting of all couples $(\alpha, \varphi(z))$ where

$$
\alpha=\left(\begin{array}{ll}
a & b \\
c & d
\end{array}\right) \in G L_{2}^{+}(\boldsymbol{R})
$$

and $\varphi$ is a holomorphic function on $H$ such that $\varphi(z)^{2}=t \cdot \operatorname{det}(\alpha)^{-1 / 2}(c z+d)$ with a complex number $t$ of absolute value 1 . The law of multiplication in $\mathcal{G}$ is defined by

$$
(\alpha, \varphi(z))(\beta, \phi(z))=(\alpha \beta, \varphi(\beta(z)) \psi(z)) .
$$

We denote by $P$ the projection map of $\mathcal{G}$ onto $G L_{2}^{+}(\boldsymbol{R})$ which sends $(\alpha, \varphi)$ to $\alpha$. For $\xi=(\alpha, \varphi) \in \mathcal{G}$, a function $f$ on $H$, and an odd integer $k$, we put

$$
\left.f\right|_{k / 2} \xi=f(\alpha(z)) \varphi(z)^{-k} .
$$

If $f \in \mathscr{M}(k / 2)$, we shall often write $\left.f\right|_{k / 2} \xi$ simply $f \mid \xi$. We can define an injection $\gamma \mapsto \gamma^{*}$ of $\Gamma_{0}(4)$ into $\mathcal{G}$ by

$$
\gamma^{*}=(\gamma, j(\gamma, z)) \quad\left(\gamma \in \Gamma_{0}(4)\right) .
$$

Our notation (1.2) shows that $\left.f\right|_{k / 2} \gamma=\left.f\right|_{k / 2} \gamma^{*}$. Another type of injection can be defined by using the well known function

$$
\eta(z)=e(z / 24) \prod_{n=1}^{\infty}(1-e(n z)) .
$$

In fact, if we put

$$
\begin{aligned}
& \eta(\gamma(z))=h(\gamma, z) \eta(z), \\
& \gamma_{*}=(\gamma, h(\gamma, z)) \quad(\gamma \in \Gamma(1)),
\end{aligned}
$$

then $\gamma \mapsto \gamma_{*}$ embeds $\Gamma(1)$ into $\mathcal{G}$. We see that $\gamma_{*}=\gamma^{*}$ for $\gamma \in \Gamma(24)$, since $\eta(z)$ belongs to $\mathcal{S}(1 / 2, \Gamma(24))$ (see [9, p. 457]).

LEMMA 1. Let $N$ be a multiple of 24 and let $\sigma \in \operatorname{Aut}(\boldsymbol{C})$. Then there is an automorphism $\gamma \mapsto \gamma^{\sigma}$ of $\Gamma(1) / \Gamma(N)$ such that: (i) $\Gamma_{0}(M) / \Gamma(N)$ is stable under $\sigma$ for every divisor $M$ of $N$; (ii) $f^{\sigma} \mid\left(\gamma^{\sigma}\right)_{*}=\left(f \mid \gamma_{*}\right)^{\sigma}$ and $f^{\sigma} \mid\left(\gamma^{\sigma}\right)^{*}=\left(f \mid \gamma^{*}\right)^{\sigma}$ for all $f \in \mathscr{M}(k / 2, \Gamma(N))$; (iii) $\gamma$ and $\gamma^{\sigma}$ have the same diagonal entries modulo $N$.

PROOF. As shown in [15, Proposition 2.1], $\mathscr{M}(k / 2, \Gamma(N))$ is spanned by $\boldsymbol{Q}$-rational elements. Therefore it is sufficient to prove the existence of an automorphism with the required property (ii) for all $\boldsymbol{Q}$-rational $f$. For this purpose we consider the action of the adele group $G_{A^{+}}$(which is the "positive part" of $\left.G L_{2}(\boldsymbol{A})\right)$ on $\boldsymbol{Q}_{\mathrm{ab}}$-rational modular functions defined in [8, Chapter 6] (see also [14], [15]). Given $\sigma$, take $t \in \Pi_{p} \boldsymbol{Z}_{p}^{\times}$so that the action of $t$ on $\boldsymbol{Q}_{\mathrm{ab}}$ coincides with $\sigma$. By the strong approximation theorem, we have 


$$
\left(\begin{array}{ll}
1 & 0 \\
0 & t
\end{array}\right) \gamma\left(\begin{array}{cc}
1 & 0 \\
0 & t^{-1}
\end{array}\right)=u \alpha
$$

with $\alpha \in S L_{2}(\boldsymbol{Q})$ and $u \in S L_{2}(\boldsymbol{A})$ such that $u_{p} \equiv 1\left(\bmod N \boldsymbol{Z}_{p}\right)$ for all primes $p$. Obviously $\alpha \in \Gamma(1)$. Given a $\boldsymbol{Q}$-rational $f$ of $\mathscr{M}(k / 2, \Gamma(N))$, put $g=f / \eta^{k}$. Then $g$ is a $\boldsymbol{Q}$-rational modular function of level $N$, so that $g^{u}=g$. Since $\left(\begin{array}{ll}1 & 0 \\ 0 & t\end{array}\right)$ acts as $\sigma^{-1}$ on modular forms, we have $(g \mid \gamma)^{\sigma}=g^{\sigma} \mid \alpha$, and hence $\left(f \mid \gamma_{*}\right)^{\sigma}=f^{\sigma} \mid \alpha_{*}$. Similarly, considering $f / \theta^{k}$ instead of $f / \eta^{k}$, we find $\left(f \mid \gamma^{*}\right)^{\sigma}=f^{\sigma} \mid \alpha^{*}$ when $\gamma \in \Gamma_{0}(4)$. Obviously $\alpha$ modulo $N$ is uniquely determined for $\gamma$ and $t$ by (2.4), Writing $\gamma^{\sigma}$ for $\alpha$, we obtain our lemma.

The above technique is also applicable to the elements of $\mathcal{G}$ whose projections do not necessarily belong to $\Gamma(1)$. For example, we can prove

LEMMA 2. For a positive integer $M$, define an operator $\xi_{M}$ acting on $M(\lambda)$ by

$$
f \mid \xi_{M}= \begin{cases}f(-1 / M z)(M z)^{-\lambda} & \text { if } \lambda \in \boldsymbol{Z}, \\ f(-1 / M z)(-i M z)^{-\lambda} & \text { if } \lambda \in \boldsymbol{Z},\end{cases}
$$

where $(-i M z)^{-\lambda}$ is chosen to be positive on the positive imaginary axis. Let $\sigma \in \operatorname{Aut}(\boldsymbol{C})$. Then, for every $f \in \mathscr{M}(\lambda, N, \chi)$, we have

$$
f^{\sigma} \mid \xi_{M}=\chi(s)^{\sigma}\left(\frac{2}{s}\right)^{2 \lambda}\left(f \mid \xi_{M}\right)^{\sigma}
$$

where $s$ is an integer such that $e(1 / 2 N)^{\sigma}=e(s / 2 N)$, and $\left(\frac{2}{s}\right)$ is the quadratic residue symbol.

Here $M$ may or may not be equal to $N$. We omit the proof, as this will be necessary only for an alternative proof of certain facts in our proof of Theorem 3.

Lemma 3. Let $X$ be a vector space over a subfield $K$ of $\boldsymbol{C}$ with countably many elements, and let $U=X \otimes_{K} C$. Let $\operatorname{Aut}(\boldsymbol{C} / K)$ denote the group of all automorphisms of $\boldsymbol{C}$ over $K$, and let $\operatorname{Aut}(\boldsymbol{C} / K)$ act on $U$ by $\left(\sum_{n} c_{n} x_{n}\right)^{\sigma}=\Sigma_{n} c_{n}^{\sigma} x_{n}$ for $\boldsymbol{\sigma} \in \operatorname{Aut}(\boldsymbol{C} / K), \quad c_{n} \in \boldsymbol{C}$, and $x_{n} \in X$. Suppose $V$ is a finite-dimensional $\boldsymbol{C}$-linear subspace of $U$ stable under $\operatorname{Aut}(\boldsymbol{C} / K)$. Then $V=(X \cap V) \otimes_{K} \boldsymbol{C}$.

Proof. We can find a finite-dimensional subspace $Y$ of $X$ such that $V \subset Y \otimes_{K} C$. Then our assertion becomes a special case of a well-known fact that every affine variety stable under $\operatorname{Aut}(\boldsymbol{C} / K)$ is $K$-rational. One can also prove our lemma in an elementary way.

LEMMA 4. For $0<\lambda \in 2^{-1} \boldsymbol{Z}$, the following assertions hold.

(1) Let $f \in \mathscr{M}(\lambda), \alpha=\left(\begin{array}{ll}a & b \\ c & d\end{array}\right) \in G L_{2}(\boldsymbol{Q})$, $\operatorname{det}(\alpha)>0$, and let $g(z)=f(\alpha(z))(c z+d)^{-\lambda}$. Then $K(g) \subset \boldsymbol{Q}_{\mathrm{ab}} K(f)$. 
(2) $\mathscr{M}(\lambda, \Gamma)$ and $\mathcal{S}(\lambda, \Gamma)$ are spanned by $\boldsymbol{Q}$-rational elements, if $\Gamma$ is either $\Gamma_{1}(N)$ or $\Gamma(N)$.

(3) $\mathscr{M}(\lambda, N, \chi)$ and $\mathcal{S}(\lambda, N, \chi)$ are spanned by $K(\chi)$-rational elements.

(4) $\mathscr{M}(\lambda, N, \chi)^{\sigma}=\mathscr{M}\left(\lambda, N, \chi^{\sigma}\right)$ and $\mathcal{S}(\lambda, N, \chi)^{\sigma}=\mathcal{S}\left(\lambda, N, \chi^{\sigma}\right)$ for every $\sigma \in \operatorname{Aut}(\boldsymbol{C})$.

Proof. If $\lambda \in \boldsymbol{Z}$, assertions (1) and (4) are included in [14, Propositions 1.4, 1.6, 1.8]. Assertion (1) for $\lambda \notin Z$ is proved in [11, Proposition 1.5] when $K(f)$ $\subset \boldsymbol{Q}_{\mathrm{ab}}$; the same proof is obviously valid even if $K(f) \leftarrow \boldsymbol{Q}_{\mathrm{ab}}$. Assertion (2) for $\mathscr{M}(\lambda, \Gamma)$ is included in [15, Proposition 2.1] if $\Gamma=\Gamma(N)$. The same proof is obviously valid for $\Gamma_{1}(N)$ also. Since $S(\lambda)$ is stable under $\operatorname{Aut}(\boldsymbol{C})$ as mentioned in $\S 1$, we obtain assertion (2) for $\mathcal{S}(\lambda, \Gamma)$ from Lemma 3. Assertion (4) for $\lambda=k / 2$ can be proved in a straightforward way by means of Lemma 1 . Then assertion (3) follows from Lemma 3.

We now recall the definition of $T\left(p^{2}\right)$. Fixing a multiple $N$ of 4 , put $\Gamma=\Gamma_{0}(N)$ and denote by $\Delta$ the image of $\Gamma$ under the map $\gamma \mapsto \gamma^{*}$. Further we consider an element $\xi$ of $\mathcal{G}$ defined by

$$
\xi=\left(\alpha, p^{1 / 2}\right), \quad \alpha=\left(\begin{array}{cc}
1 & 0 \\
0 & p^{2}
\end{array}\right)
$$

with a prime $p$ and then a coset decomposition

$$
\Gamma \alpha \Gamma=\amalg_{\nu} \Gamma \alpha_{\nu}, \quad \alpha_{\nu}=\left(\begin{array}{ll}
a_{\nu} & b_{\nu} \\
c_{\nu} & d_{\nu}
\end{array}\right) .
$$

As shown in $[\mathbf{9}]$, we have $\Delta \xi \Delta=\amalg_{\nu} \Delta \xi_{\nu}$ with elements $\xi_{\nu}$ such that $P\left(\xi_{\nu}\right)=\alpha_{\nu}$. Now, for $f \in \mathscr{M}(k / 2, N, \chi)$, we put

$$
f\left|T_{N}\left(p^{2}\right)=p^{(k / 2)-2} \sum_{\nu} \chi\left(a_{\nu}\right) f\right| \xi_{\nu} .
$$

Lemma 5. Let $p$ be a prime not dividing $N$. Then

$$
\chi\left(p^{2}\right)\left\langle f \mid T\left(p^{2}\right), g\right\rangle=\left\langle f, g \mid T\left(p^{2}\right)\right\rangle
$$

for every $f, g \in \mathcal{S}(k / 2, N, \chi)$.

Proof. Observe that

$$
\langle f \mid \delta, g\rangle=\left\langle f, g \mid \delta^{-1}\right\rangle \quad \text { for every } \delta \in \mathcal{G} .
$$

Therefore

$$
\left\langle f \mid T\left(p^{2}\right), g\right\rangle=p^{(k / 2)-2}\left\langle\sum_{\nu} \chi\left(a_{\nu}\right) f \mid \xi_{\nu}, g\right\rangle=p^{(k / 2)-2}\left\langle f, \sum_{\nu} \chi\left(a_{\nu}\right)^{-1} g \mid \xi_{\nu}^{-1}\right\rangle .
$$

By [8, Lemma 3.5], we can choose $\alpha_{\nu}$ so that $\Gamma \alpha \Gamma=\amalg_{\nu} \Gamma \alpha_{\nu}=\amalg_{\nu} \alpha_{\nu} \Gamma$. Then $\Delta \xi^{-1} \Delta=\amalg_{\nu} \Delta \xi_{\nu}^{-1}$. Put

$$
\eta=\left(\left(\begin{array}{ll}
p^{2} & 0 \\
0 & 1
\end{array}\right), p^{-1 / 2}\right), \quad \zeta=\left(p^{2}\left(\begin{array}{ll}
1 & 0 \\
0 & 1
\end{array}\right), 1\right) .
$$


As shown in the proof of [9, Theorem 1.7], we have $\xi^{-1} \zeta=\eta \in \Delta \xi \Delta$, and hence $\Delta \xi \Delta=\Delta \xi^{-1} \zeta \Delta=\amalg_{\nu} \Delta \xi_{\nu}^{-1 \zeta}$. Since $P\left(\xi_{\nu}^{-1} \zeta\right)=\left(\begin{array}{ll}d_{\nu} & * \\ * & a_{\nu}\end{array}\right)$, we have

$$
?\left|T\left(p^{2}\right)=p^{(k / 2)-2} \sum_{\nu} \chi\left(d_{\nu}\right) g\right| \xi_{\nu}^{-1}=p^{(k / 2)-2} \chi\left(p^{2}\right) \sum_{\nu} \chi\left(a_{\nu}\right)^{-1} g \mid \xi_{\nu}^{-1},
$$

which proves our assertion.

This shows that the operators $\chi(p)^{-1} T\left(p^{2}\right)$ for $p$ prime to $N$ are hermitian on $\mathcal{S}(k / 2, N, \chi)$; since they commute, we can find an orthogonal basis of $\mathcal{S}(k / 2, N, \chi)$ consisting of eigenforms for all $p$ prime to $N$. We also observe that $\mathcal{S}(k / 2, N, \chi, F)$ is orthogonal to $\mathcal{S}\left(k / 2, N, \chi, F^{\prime}\right)$ if $F \neq F^{\prime}$.

LEMma 6. Suppose $k$ is an odd integer $>1$. If $\mathcal{S}(k / 2, N, \chi, F) \neq\{0\}$ with a primitive $F$, then $F$ belongs to $\mathcal{S}\left(k-1, N^{\prime}\right)$ with a divisor $N^{\prime}$ of a power of $N$. Moreover, if $F \mid T(p)=c(p) F$ for $p \nmid N$, then $f \mid T\left(p^{2}\right)=c(p) f$ for all $f \in$ $\mathcal{S}(k / 2, N, \chi, F)$ and all $p \nmid N$.

Proof. As remarked above, $\mathcal{S}(k / 2, N, \chi)$ has an orthogonal basis $\left\{g_{1}, \cdots, g_{n}\right\}$ consisting of eigenfunctions of $T_{N}\left(p^{2}\right)$ for all $p$ prime to $N$. We take it so that $g_{1}, \cdots, g_{m} \in \mathcal{S}(k / 2, N, \chi, F)$ and $g_{m+1}, \cdots, g_{n} \notin \mathcal{S}(k / 2, N, \chi, F)$. We see easily that no nontrivial linear combination of $g_{m+1}, \cdots, g_{n}$ belongs to $\mathcal{S}(k / 2, N, \chi, F)$. Therefore $g_{1}, \cdots, g_{m}$ span $\mathcal{S}(k / 2, N, \chi, F)$. Let $g_{1} \mid T\left(p^{2}\right)=b(p) g_{1}$ for $p \nmid N$, and let $S_{1}$ be the set of all elements $f$ of $\mathcal{S}(k / 2, N, \chi)$ such that $f \mid T\left(p^{2}\right)=b(p) f$ for all $p \nmid N$. Since $S_{1}$ is stable under $T_{N}\left(p^{2}\right)$ for all $p$, it contains an eigenfunction $f_{0}$ of $T_{N}\left(p^{2}\right)$ for all $p$; let $f_{0} \mid T_{N}\left(p^{2}\right)=b(p) f_{0}$ for every $p$ (including the prime factors of $N)$. The main result of [9] shows that there is an element $h$ of $\mathscr{M}\left(k-1, N^{\prime}, \chi^{2}\right)$ such that $D(s, h)$ is given by (1.11), where $N^{\prime}$ can be taken to be a multiple of $N$ which divides a power of $N ; h \in \mathcal{S}(k-1)$ if $k>3$. Since $D(s, h)$ has an Euler product, $h$ is an eigenfunction of all Hecke operators of level $N^{\prime}$; in particular $h \mid T(p)=b(p) h$ for all $p \nmid N$. Since $F \mid T(p)=b(p) F$ for almost all $p$, we see that $h$ is a cusp form even when $k=3$. Therefore the " exact level" of $F$ divides $N^{\prime}$, and hence $F \mid T(p)=b(p) F$ for all $p \nmid N$. Applying this result also to $g_{2}, \cdots, g_{m}$, we obtain our assertion. Notice that if $k>3$, the same reasoning shows that each of $g_{m+1}, \cdots, g_{n}$ belongs to $\mathcal{S}\left(k / 2, N, \chi, F^{\prime}\right)$ with some $F^{\prime}$.

We mention here Shintani [16], Niwa [4], Gelbart and Piatetski-Shapiro [2], and Flicker [1] as investigations related to the correspondence between $\mathcal{S}(k / 2)$ and $\mathcal{S}(k-1)$.

LEMMA 7. (1) $\mathcal{S}(k / 2, N, \chi, F)$ is spanned by $K(\chi, F)$-rational elements.

(2) $\mathcal{S}(k / 2, N, \chi, F)^{\sigma}=\mathcal{S}\left(k / 2, N, \chi^{\sigma}, F^{\sigma}\right)$ for every $\sigma \in \operatorname{Aut}(\boldsymbol{C})$.

(3) Let $\mathscr{I}(k / 2, N, \chi, F)$ be the orthogonal complement of $\mathcal{S}(k / 2, N, \chi, F)$ in $\mathcal{S}(k / 2)$. Then $\mathscr{I}(k / 2, N, \chi, F)^{\sigma}=\mathfrak{I}\left(k / 2, N, \chi^{\sigma}, F^{\sigma}\right)$.

(4) If $k \geqq 5, \mathcal{S}(k / 2, N, \chi)$ is the direct sum of subspaces $\mathcal{S}(k / 2, N, \chi, F)$ for 
finitely many $F$ 's.

Proof. We first observe that

$$
\left(f \mid T\left(p^{2}\right)\right)^{\sigma}=f^{\sigma} \mid T\left(p^{2}\right) .
$$

This follows from [9, Theorem 1.7] and Lemma 4, (4). Now take $g_{1}, \cdots, g_{n}$ as in the proof of Lemma 6 for a fixed $F$. Put

$$
\mathscr{I}_{0}(k / 2, N, \chi, F)=\mathcal{S}(k / 2, N, \chi) \cap \mathscr{I}(k / 2, N, \chi, F) .
$$

Then $\mathscr{I}_{0}(k / 2, N, \chi, F)$ is spanned by $g_{m+1}, \cdots, g_{n}$. By $(2.7), g_{1}^{\sigma}, \cdots, g_{n}^{\sigma}$ are eigenfunctions of $T\left(p^{2}\right)$ for all $p$ prime to $N$ in $\mathcal{S}\left(k / 2, N, \chi^{\sigma}\right)$, and obviously $g_{1}^{\sigma}, \cdots, g_{m}^{\sigma}$ belong to $\mathcal{S}\left(k / 2, N, \chi^{\sigma}, F^{\sigma}\right)$. Let $F \mid T(p)=c(p) F$. If $i>m$, we have $g_{i} \mid T\left(p^{2}\right)=c^{\prime}(p) g_{i}$ with $c^{\prime}(p) \neq c(p)$ for infinitely many $p$. Then, by $(2.7), g_{i}^{\sigma}$ does not belong to $\mathcal{S}\left(k / 2, N, \chi^{\sigma}, F^{\sigma}\right)$; moreover, $g_{i}^{\sigma}$ is orthogonal to $\mathcal{S}\left(k / 2, N, \chi^{\sigma}, F^{\sigma}\right)$ by virtue of Lemma 5 , Therefore $g_{m+1}^{\sigma}, \cdots, g_{n}^{\sigma}$ span $\mathscr{I}_{0}\left(k / 2, N, \chi^{\sigma}, F^{\sigma}\right)$. This proves (2) and $\mathscr{I}_{0}(k / 2, N, \chi, F)^{\sigma}=\mathscr{I}_{0}\left(k / 2, N, \chi^{\sigma}, F^{\sigma}\right)$. Then (1) follows from Lemma 3, Assertion (4) follows from the remark at the end of the proof of Lemma 6. To complete the proof of (3), denote by $\mathcal{U}(k / 2, M, \chi)$ the orthogonal complement of $\mathcal{S}(k / 2, N, \chi)$ in $\mathcal{S}(k / 2, \Gamma(M))$ for every multiple $M$ of $N$. Obviously $\mathscr{I}(k / 2, N, \chi, F)$ is the sum of $I_{0}(k / 2, N, \chi, F)$ and $U(k / 2, M, \chi)$ for all multiples $M$ of $N$. Thus our task is to show that $U(k / 2, M, \chi)^{\sigma}=$ $\mho\left(k / 2, M, \chi^{\sigma}\right)$. Observe that $U(k / 2, M, \chi)$ is stable under $\Gamma_{0}(N)$. Taking a basis $\left\{f_{1}, \cdots, f_{m}\right\}$ of $U(k / 2, M, \chi)$, we can define a representation $\left(a_{i j}(\gamma)\right)$ of $\Gamma_{0}(N)$ (or rather, of $\Gamma_{0}(N) / \Gamma(M)$ ) by $f_{i} \mid \gamma=\Sigma_{j} a_{i j}(\gamma) f_{j}$. Since this does not contain the representation $\gamma \mapsto \chi\left(d_{\gamma}\right)$, we have

$$
\sum_{\gamma \in R} \chi\left(d_{\gamma}\right)^{\rho} a_{i j}(\gamma)=0 \quad(i, j=1, \cdots, m),
$$

where $R=\Gamma_{0}(N) / \Gamma(M)$, and $\rho$ denotes the complex conjugation. Given $\sigma \in \operatorname{Aut}(\boldsymbol{C})$, let $\gamma \mapsto \gamma^{\sigma}$ be the automorphism of $\Gamma_{0}(N) / \Gamma(M)$ of Lemma 1. If $h \in \mathcal{S}\left(k / 2, N, \chi^{\sigma}\right)$, we have

$$
\begin{aligned}
{\left[\Gamma_{0}(N): \Gamma(M)\right]\left\langle h, f_{i}^{\sigma}\right\rangle } & =\sum_{\gamma \in R} \chi\left(d_{\gamma}\right)^{\sigma \rho} \chi\left(d_{\gamma}\right)^{\sigma}\left\langle h, f_{i}^{\sigma}\right\rangle \\
& =\sum_{\gamma \in R} \chi\left(d_{\gamma}\right)^{\sigma \rho}\left\langle h \mid\left(\gamma^{\sigma}\right)^{-1}, f_{i}^{\sigma}\right\rangle=\sum_{\gamma \in R} \chi\left(d_{\gamma}\right)^{\rho \sigma}\left\langle h, f_{i}^{\sigma} \mid \gamma^{\sigma}\right\rangle \\
& =\sum_{j} \sum_{\gamma \in R} \chi\left(d_{\gamma}\right)^{\rho \sigma} a_{i j}(\gamma)^{\sigma}\left\langle h, f_{j}^{\sigma}\right\rangle=0 .
\end{aligned}
$$

Therefore $f_{1}^{\sigma}, \cdots, f_{m}^{\sigma}$ belong to $\mathcal{U}\left(k / 2, M, \chi^{\sigma}\right)$. This completes the proof.

Lemma 8. Suppose $0 \neq f \in \mathcal{S}(k / 2, N, \chi)$. Then $K(\chi) \subset K(f)$. If moreover $f \in \mathcal{S}(k / 2, N, \chi, F)$, then $K(\chi, F) \subset K(f)$.

This follows from (4) of Lemma 4 and (2) of Lemma 7. 


\section{Eisenstein series and differential operators.}

LeMma 9. Let $g$ be a $\boldsymbol{C}$-valued continuous function on $H$ such that $\left.g\right|_{k / 2} \gamma=g$ for every $\gamma \in \Gamma$ with a congruence subgroup $\Gamma$ of $\Gamma_{0}(4)$. Suppose that $\langle f, g\rangle$ defined by (1.5) is meaningful for all $f \in \mathcal{S}(k / 2)$. Then there is an element $h$ of $\mathcal{S}(k / 2, \Gamma)$ uniquely determined by $g$, such that $\langle f, g\rangle=\langle f, h\rangle$ for all $f \in \mathcal{S}(k / 2, \Gamma)$. Moreover this $h$ satisfies $\langle f, g\rangle=\langle f, h\rangle$ for all $f \in \mathcal{S}(k / 2)$.

We call $h$ the essential part of $g$.

Proof. The first assertion is obvious. Take $N$ so that $\Gamma(N) \subset \Gamma$. Then there exists an element $p$ of $\mathcal{S}(k / 2, \Gamma(N))$ such that $\langle f, g\rangle=\langle f, p\rangle$ for all $f \in \mathcal{S}(k / 2, \Gamma(N))$. Let $\gamma \in \Gamma$. Then $\mathcal{S}(k / 2, \Gamma(N))$ is stable under $f \mapsto f \mid \gamma$, and therefore

$$
\langle f, p \mid \gamma\rangle=\left\langle f \mid \gamma^{-1}, p\right\rangle=\left\langle f \mid \gamma^{-1}, g\right\rangle=\langle f, g \mid \gamma\rangle=\langle f, g\rangle=\langle f, p\rangle
$$

for all $f \in \mathcal{S}(k / 2, \Gamma(N))$. Hence $p \mid \gamma=p$, i. e., $p \in \mathcal{S}(k / 2, \Gamma)$. By the uniqueness of $h$, we have $h=p$, which proves our lemma.

REMARK. The same type of result obviously holds for the forms of integral weight, and even for modular forms on a higher-dimensional domain. In fact, it is a purely algebraic statement concerning a bilinear form on a vector space on which a system of groups acts under some conditions.

For a congruence subgroup $\Gamma$ of $\Gamma_{0}(4)$, define now a subspace $\mathcal{E}(k / 2, \Gamma)$ of $\mathscr{M}(k / 2, \Gamma)$ by

$$
\mathcal{E}(k / 2, \Gamma)=\{g \in \mathscr{M}(k / 2, \Gamma) \mid\langle g, f\rangle=0 \text { for all } f \in \mathcal{S}(k / 2, \Gamma)\} .
$$

Obviously $\mathscr{M}(k / 2, \Gamma)=\mathcal{S}(k / 2, \Gamma) \oplus \mathcal{E}(k / 2, \Gamma)$. Moreover, taking $h=0$ and $g$ in $\mathcal{E}(k / 2, \Gamma)$ in the above lemma, we find that

$$
\mathcal{E}(k / 2, \Gamma)=\mathscr{M}(k / 2, \Gamma) \cap \mathcal{E}\left(k / 2, \Gamma^{\prime}\right) \quad \text { if } \quad \Gamma^{\prime} \subset \Gamma .
$$

Lemma 10. Suppose $k \neq 3$. Then $\mathcal{E}\left(k / 2, \Gamma_{1}(N)\right)$ is spanned by its Q-rational elements.

PROOF. If $k=1$, this is a direct consequence of a result of Serre and Stark [7, Theorem 6]. Suppose $k>3$. Let us first prove our assertion for $\Gamma(N)$ instead of $\Gamma_{1}(N)$. Fix a positive integer $N>2$ and denote by $R$ a complete set of representatives of $\Gamma_{N, \infty} \backslash \Gamma(N)$, where

$$
\Gamma_{N, \infty}=\left\{\left(\begin{array}{cc}
1 & m N \\
0 & 1
\end{array}\right) \mid m \in \boldsymbol{Z}\right\} .
$$

Define $h(\gamma, z)$ by $(2.3)$ and put

$$
G(z)=\sum_{\gamma \in R} h(\gamma, z)^{-k},
$$




$$
G_{\alpha}(z)=G(\alpha(z)) h(\alpha, z)^{-k} \quad(\alpha \in \Gamma(1)) .
$$

Also denote by $\mathscr{M}_{N}^{\prime}$ the set of all elements $f$ of $\mathscr{M}(k / 2)$ such that $f(\gamma(z))=$ $h(\gamma, z)^{k} f$ for every $\gamma \in \Gamma(N)$, and put $\mathcal{S}_{N}^{\prime}=\mathscr{M}_{N}^{\prime} \cap \mathcal{S}(k / 2)$. Now a standard argument (originally due to Petersson) shows that $\mathcal{S}_{N}^{\prime}$ is orthogonal to $G$, and hence to $G_{\alpha}$ for all $\alpha \in \Gamma(1)$. Moreover $G_{\alpha}$ vanishes at all cusps of $\Gamma(N)$ except at $\alpha^{-1}(\infty)$; therefore every element of $\mathscr{M}_{N}^{\prime}$ is a linear combination of $G_{\alpha}$ plus a cusp form. (For details, see Petersson [5, Satz 2], [6].) Therefore if we put

$$
\mathcal{E}_{N}^{\prime}=\left\{f \in \mathscr{M}_{N}^{\prime} \mid\left\langle f, \mathcal{S}_{N}^{\prime}\right\rangle=0\right\},
$$

then $\mathcal{E}_{N}^{\prime}$ is spanned by $G_{\alpha}$ for all $\alpha \in \Gamma(1)$. Suppose now $24 \mid N$, and put, for every character $\omega$ modulo $N$,

$$
\begin{aligned}
& G(z, \omega)=\sum_{\gamma \in W} \omega\left(d_{\gamma}\right) j(\gamma, z)^{-k}, \\
& G^{*}(z, \omega)=G(-1 / z, \omega)(-i z)^{-k / 2},
\end{aligned}
$$

where $W$ is a complete set of representatives of $\Gamma_{1, \infty} \backslash \Gamma_{0}(N)$. Obviously (3.3a) vanishes if $\omega(-1)=-1$; so we consider only those characters $\omega$ satisfying $\omega(-1)=1$. Observe that the sum of $G(z, \omega)$ for all such $\omega$ is a positive integer times $G$. Now the Fourier expansion of $G^{*}$ was studied in [10]. In fact,

$$
G^{*}(z, \omega)=2 e(k / 8) N^{-k / 2} E^{*}(z / N, 0,-k, \omega)
$$

with the function $E^{*}$ of $[10$, p. 86$]$. As shown on $[10$, p. 89$]$, if we put

$$
G^{*}(z, \omega)=\sum_{n=1}^{\infty} B(n, \omega) e(n z / N)
$$

and $n=t m^{2}$ with a square-free $t$, then

$$
\begin{aligned}
& N^{k / 2} \Gamma(k / 2) L_{N}\left(k-1, \omega^{2}\right) B(n, \omega) \\
& =2 n^{(k / 2)-1}(2 \pi)^{k / 2} L_{N}\left(\lambda, \omega_{t}\right) \sum_{a, b} \mu(a) \omega_{t}(a) \omega(b)^{2} a^{-\lambda} b^{2-k},
\end{aligned}
$$

where $\lambda=(k-1) / 2, \mu$ is the Moebius function, $\omega_{t}$ is a primitive character such that $\omega_{t}(a)=\left(\frac{-1}{a}\right)^{\lambda}\left(\frac{t N}{a}\right) \omega(a)$ for $(a, t N)=1$, and the sum $\sum_{a, b}$ is extended over all positive integers $a, b$ prime to $N$ such that $a b$ divides $m$. For Dirichlet characters $\chi_{1}, \cdots, \chi_{n}$, put

$$
\left[\chi_{1}, \cdots, \chi_{n}\right]=\tau\left(\chi_{1}\right) \cdots \tau\left(\chi_{n}\right) / \tau\left(\chi_{1} \cdots \chi_{n}\right),
$$

$$
P_{N}\left(n, \chi_{1}\right)=(2 \pi i)^{-n} \tau\left(\chi_{1}\right)^{-1} L_{N}\left(n, \chi_{1}\right) \text { for } 0<n \in \boldsymbol{Z}, \chi_{1}(-1)=(-1)^{n} \text {. }
$$

It can easily be shown that, for every $\sigma \in \operatorname{Aut}(\boldsymbol{C})$,

$$
\left[\chi_{1}, \cdots, \chi_{m}\right]^{\sigma}=\left[\chi_{1}^{\sigma}, \cdots, \chi_{m}^{\sigma}\right] \text {, }
$$




$$
P_{N}\left(n, \chi_{1}\right)^{\sigma}=P_{N}\left(n, \chi_{1}^{\sigma}\right)
$$

(see $\left[14\right.$, Lemma 4.12, Proposition 3.1]). Putting $\varphi_{t}(a)=\left(\frac{-1}{a}\right)^{\lambda}\left(\frac{t N}{a}\right)$, we see that

$$
\begin{aligned}
& \sqrt{2} \tau(\omega) P_{N}\left(2 \lambda, \omega^{2}\right) B(n, \omega) \\
& =A(k, N, n)[\omega, \omega]\left[\varphi_{t}, \omega\right]^{-1} P_{N}\left(\lambda, \omega_{t}\right) \sum_{a, b} \mu(a) \omega_{t}(a) \omega(b)^{2} a^{-\lambda} b^{2-k}
\end{aligned}
$$

with a rational number $A(k, N, n)$ depending only on $k, N, n$. This combined with $(3.6 \mathrm{a}, \mathrm{b})$ shows that

$$
\left[\sqrt{2} \tau(\omega) G^{*}(z, \omega)\right]^{\sigma}=\sqrt{2} \tau\left(\omega^{\sigma}\right) G^{*}\left(z, \omega^{\sigma}\right)
$$

for every $\sigma \in \operatorname{Aut}(\boldsymbol{C})$. Now $G(z, \omega)$ belongs to $\mathscr{M}(k / 2, N)$; again a standard argument shows that it is orthogonal to $\mathcal{S}(k / 2, N, \chi)$ for every $\chi$, and hence it belongs to $\mathcal{E}\left(k / 2, \Gamma_{1}(N)\right)$. Therefore $G^{*}(z, \omega)$ belongs to $\mathcal{E}(k / 2, \Gamma(N))$. Since $\mathcal{E}_{N}^{\prime}=\mathcal{E}(k / 2, \Gamma(N))$ if $24 \mid N$, this shows that $\mathcal{E}(k / 2, \Gamma(N))$ is generated by $G^{*}(\gamma(z), \omega) h(\gamma, z)^{-k}$ for all $\gamma \in \Gamma(1)$ and all $\omega$. This combined with Lemma 1 and (3.7) shows that $\mathcal{E}(k / 2, \Gamma(N))$ is stable under $\operatorname{Aut}(\boldsymbol{C})$. So far we have assumed $24 \mid N$. Given an arbitrary multiple $M$ of 4 , let $N$ be a common multiple of 24 and $M$. By (3.1), we have

$$
\mathcal{E}\left(k / 2, \Gamma_{1}(M)\right)=\mathscr{M}\left(k / 2, \Gamma_{1}(M)\right) \cap \mathcal{E}(k / 2, \Gamma(N)),
$$

and hence $\mathcal{E}\left(k / 2, \Gamma_{1}(M)\right)$ is stable under $\operatorname{Aut}(\boldsymbol{C})$. Therefore it has a $\boldsymbol{Q}$-rational basis by virtue of Lemma 3 ,

The corresponding fact for all integral weights $\geqq 1$ can be derived by the same (and actually easier) argument from the well known results of Hecke on Eisenstein series. It is conjecturable that the above lemma is true also for $k=3$.

We now introduce a differential operator $\delta_{t}$ and $\delta_{t}^{r}$ for $t \in \boldsymbol{C}$ and $0 \leqq r \in \boldsymbol{Z}$ by

$$
\begin{aligned}
& \delta_{t}^{r}=\delta_{t+2 r-2} \cdots \delta_{t+2} \delta_{t}, \\
& \delta_{t}=(2 \pi i)^{-1}\left[t(2 i y)^{-1}+(\partial / \partial z)\right] \quad \text { (cf. [12], [14]). }
\end{aligned}
$$

We see easily that

$$
\delta_{\lambda}^{r}\left(\left.f\right|_{\lambda} \gamma\right)=\left.\left(\delta_{\lambda}^{r} f\right)\right|_{\lambda+2 r} \gamma . \quad\left(\lambda \in 2^{-1} Z, \gamma \in \Gamma_{0}(4)\right) .
$$

Let $\mathcal{A}_{\lambda}$ denote the set of all functions $g$ on $H$ of the form

$$
g=\sum_{0 \leq r \geqq \lambda / 2} \delta_{\lambda-2 r}^{r} g_{r} \quad \text { with } \quad g_{r} \in \bigcup_{N=1}^{\infty} \mathscr{M}(\lambda-2 r, N) .
$$

Then the following facts can easily be proved (see [12]) :

The $g_{r}$ are unique for $g$.

$$
\text { If } f \in \mathcal{A}_{\lambda} \text { and } g \in \mathcal{A}_{\mu} \text {, then } f g \in \mathcal{A}_{\lambda+\mu}
$$


For $\sigma \in \operatorname{Aut}(\boldsymbol{C})$ and $g$ as in (3.9), we define $g^{\sigma}$ by

$$
g^{\sigma}=\sum_{r} \delta_{\lambda-2 r}^{r} g_{r}^{\sigma} .
$$

This can be defined also as follows: $g$ has an expression

$$
g(z)=\sum_{0 \leqq s \leq \lambda / 2}(-4 \pi y)^{-s} \sum_{n=0}^{\infty} c_{s}(n) e(n z)
$$

with $c_{s}(n) \in \boldsymbol{C}$; then

$$
g^{\sigma}(z)=\sum_{s}(-4 \pi y)^{-s} \sum_{n=0}^{\infty} c_{s}(n)^{\sigma} e(n z)
$$

Therefore we have $(f g)^{\sigma}=f^{\sigma} g^{\sigma}$ for two such functions $f$ and $g$.

LEMma 11. Let $f \in \mathcal{S}(\lambda)$ and $g \in \mathscr{M}(\lambda-2 r)$ with $0\left\langle r \in Z\right.$. Then $\left\langle f, \delta_{\lambda-2 r}^{r} g\right\rangle=0$.

PROOF. It is sufficient to prove the case where $f \in \mathcal{S}(\lambda, N, \chi)$ and $g \in \mathscr{M}(\lambda-2 r, N, \chi)$. If $\lambda \in Z$, this was proved in [12]. The case of half-integral weight can be proved in exactly the same fashion.

LEMma 12. For every $g \in \mathcal{A}_{\lambda}$, let $[g]$ denote the essential part of $g$ in the sense of Lemma 9. Suppose $\lambda \neq 3 / 2$. Then $[g]^{\sigma}=\left[g^{\sigma}\right]$ for every $\sigma \in \operatorname{Aut}(\boldsymbol{C})$.

Proof. Write $g$ in the form (3.9), and let $g_{0}=p+q$ with $p \in \mathcal{S}(\lambda, N)$ and $q \in \mathcal{E}\left(\lambda, \Gamma_{1}(N)\right)$ for some $N$. Then $p=[g]$ by virtue of Lemma 11. If we change $g$ for $g^{\sigma}$, then naturally $g_{0}$ is changed into $g_{0}^{\sigma}=p^{\sigma}+q^{\sigma}$. We know that $p^{\sigma} \in \mathcal{S}(\lambda)$, and also, by Lemma 10, that $q^{\sigma} \in \mathcal{E}\left(\lambda, \Gamma_{1}(N)\right.$ ) (though, strictly speaking, we proved only the case $\lambda \notin Z$ ). Therefore we obtain our assertion.

\section{Proof of Theorem 1 .}

Let $k$ and $l$ be odd integers such that $0<l<k$, and let $f \in \mathcal{S}(k / 2, M, \chi)$ and $g \in \mathscr{M}\left(l / 2, M^{\prime}, \phi\right)$ with Fourier expansions

$$
f(z)=\sum_{n=1}^{\infty} a(n) e(n z), \quad g(z)=\sum_{n=0}^{\infty} b(n) e(n z) .
$$

For a typographical reason, let us denote the function $f^{\rho}$ defined by (1.7) also by $f_{\rho}$. By Lemma $4, f_{\rho} \in \mathcal{S}\left(k / 2, M, \chi^{-1}\right)$. Now a straightforward calculation shows that

$$
\int_{0}^{\infty} y^{s-1} \int_{0}^{1} \overline{f_{\rho}(z)} g(z) d x d y=\Gamma(s)(4 \pi)^{-s} D(s, f, g)
$$

for sufficiently large $\operatorname{Re}(s)$. The left-hand side can be transformed, by a well known principle (see [9], [12], for example), to the form

$$
\int_{\Phi} \bar{f}_{\rho} g \cdot E_{\lambda, N}(z, s+1-(k / 2), \omega) y^{s+1} d \mu(z)
$$


where $\Phi=\Gamma_{0}(N) \backslash H, N$ is any common multiple of $M$ and $M^{\prime}, \lambda=(k-l) / 2$, and

$$
\begin{aligned}
& E_{\lambda, N}(z, s, \omega)=\sum_{c, d} \omega(d)(c z+d)^{-\lambda}|c z+d|^{-2 s}, \\
& \omega(d)=\left(\frac{-1}{d}\right)^{\lambda} \chi(d) \phi(d) ;
\end{aligned}
$$

$(c, d)$ runs over the lower entries of the elements of a complete set of representatives of $\left[\{ \pm 1\} \Gamma_{1, \infty}\right] \backslash \Gamma_{0}(N) ; \Gamma_{1, \infty}$ is defined by (3.2), It is well known that

$$
\Gamma(s+\lambda) L_{N}(2 s+\lambda, \omega) E_{\lambda, N}(z, s, \omega)
$$

can be continued to an entire function on the whole plane. Moreover, integral (4.2) multiplied by $\Gamma(s+\lambda) L_{N}(2 s+\lambda, \omega)$ is convergent by virtue of [9, Lemma 3.3], and therefore defines an entire function. This proves the first part of Theorem 2 .

To prove Theorem 1, we specialize the above consideration to the case where $l=1$ and

$$
g(z)=(1 / 2) \sum_{n=-\infty}^{\infty} \varphi(n) e\left(t n^{2} z\right)
$$

with a primitive character $\varphi$ modulo $r$ such that $\varphi(-1)=1$ and a positive integer $t$. By $\left[9\right.$, Propositions 1.3 and 2.2], $g$ belongs to $\mathscr{M}\left(1 / 2,4 t r^{2}, \psi\right)$ with $\psi(m)=$ $\varphi(m)\left(\frac{t}{m}\right)$. The above computation specialized to this case shows that

$$
\begin{aligned}
& \Gamma(s)(4 \pi)^{-s} t^{-s} \sum_{n=1}^{\infty} \varphi(n) a\left(t n^{2}\right) n^{-2 s} \\
& =\int_{\Phi} \bar{f}_{\rho} g \cdot E_{\lambda, N}(z, s+1-(k / 2), \omega) y^{s+1} d \mu(z), \\
& \lambda=(k-1) / 2, \quad \omega=\varphi_{t} \chi \varphi, \quad \varphi_{t}(m)=\left(\frac{-1}{m}\right)^{\lambda}\left(\frac{t}{m}\right) .
\end{aligned}
$$

Suppose $\mathcal{S}(k / 2, M, \chi, F) \neq\{0\}$ and $F \mid T(p)=c(p) F$ for each prime $p$. By Lemma 6, we have $f \mid T\left(p^{2}\right)=c(p) f$ for all $f \in \mathcal{S}(k / 2, M, \chi, F)$ and for all $p \nmid M$. We can also find the above character $\varphi$ so that

$$
\begin{aligned}
& \text { every prime factor of } M \text { divides } r \text {, } \\
& g \text { is a cusp form. }
\end{aligned}
$$

For example, let $p_{1}, \cdots, p_{n}$ be the odd prime factors of $M$, and put

$$
\varphi(d)=\left(\frac{r}{d}\right), \quad r=8 p_{1} \cdots p_{n} q
$$

with an odd prime $q$ such that $q \equiv 3(\bmod 4)$ and $q \nmid M$. Then $\varphi$ is not totally 
even in the sense of Serre and Stark [7, p.36], and therefore $g$ is a cusp form by virtue of $[7$, Theorem $\mathrm{B}]$. Now take $t$ under the condition

$$
t \text { has no square factor prime to } r \text {. }
$$

Let $0 \neq f \in \mathcal{S}(k / 2, M, \chi, F)$. With a fixed $t$, let $N$ be the least common multiple of $M$ and $t r^{2}$. Then it follows from [9, Corollary 1.8, Theorem 1.9] that

$$
\sum_{n=1}^{\infty} \varphi(n) a\left(t n^{2}\right) n^{-2 s}=a(t) L_{N}(2 s+1-\lambda, \omega)^{-1} D(2 s, F, \varphi) .
$$

Put $m=k-2$ and evaluate (4.3) at $s=m / 2$. Then we have

$$
a(t) D(m, F, \varphi)=R \pi^{(m+1) / 2} t^{1 / 2} L_{N}(\lambda, \omega)\left\langle f^{\rho}, g \cdot E_{\lambda, N}(z, \omega)\right\rangle,
$$

where $R$ is a positive rational number which depends only on $m, N$, and $t$, and is independent of $f$, and

$$
E_{\lambda, N}(z, \omega)=E_{\lambda, N}(z, 0, \omega) .
$$

If $\lambda \neq 2$ or $\omega$ is non-trivial, $E_{\lambda, N}(z, \omega)$ belongs to $\mathscr{M}\left(\lambda, N, \omega^{-1}\right)$ and therefore $g \cdot E_{\lambda, N}(z, \omega) \in \mathcal{S}\left(k / 2, N, \chi^{-1}\right)$. If $\lambda=2$ (and hence $k=5$ ) and $\omega$ is trivial, $g \cdot E_{2, N}(z, \omega)$ belongs to $\mathcal{A}_{5 / 2}$ for the same reason as on [12, p. 795], and therefore

$$
g \cdot E_{2, N}(z, \omega)=g_{0}+\delta_{1 / 2} g_{1}
$$

with $g_{0} \in \mathscr{M}\left(5 / 2, N, \chi^{-1}\right)$ and $g_{1} \in \mathscr{M}\left(1 / 2, N, \chi^{-1}\right)$. Since $g$ is a cusp form, we see that both $g_{0}$ and $g_{1}$ are cusp forms. (Actually $g_{1}$ is a constant multiple of g.) Put $g_{0}=g \cdot E_{\lambda, N}(z, \omega)$ if $\lambda \neq 2$ or $\omega$ is nontrivial, and let $g_{0}=h+h^{\prime}$ with

$$
h \in \mathcal{S}\left(k / 2, M, \chi^{\rho}, F^{\rho}\right), \quad h^{\prime} \in \mathscr{I}\left(k / 2, M, \chi^{\rho}, F^{\rho}\right),
$$

where $\mathscr{I}$ is defined in Lemma 7, Observing that $i^{\lambda} t^{1 / 2} \tau\left(\varphi_{t}\right)$ is a rational number, we obtain, in view of (1.8),

$$
R^{\prime} a(t) A(m, F, \varphi)=\left[\varphi_{t}, \chi, \varphi\right]^{-1} P_{N}(\lambda, \omega) \pi i \cdot \tau(\chi)\left\langle h^{o}, f\right\rangle,
$$

where $R^{\prime}$ is a nonzero rational number independent of $f$.

Since $\lambda \geqq 1$, we have $P_{N}(\lambda, \omega) \neq 0$. Suppose now $k \geqq 5$. Then we have $m \geqq k / 2$, and hence $D(m, F, \varphi) \neq 0$ by [14, Proposition 4.16] (cf. also [12, Proposition 2]). Write $a(t, f)$ for a(t) in order to emphasize the dependence on $f$. Then the above relation shows that

$$
a(t, f)=0 \text { if and only if }\left\langle h^{\rho}, f\right\rangle=0 .
$$

Let $\sigma \in \operatorname{Aut}(\boldsymbol{C})$, and take $f^{\sigma}, F^{\sigma}$, and $\varphi^{\sigma}$ instead of $f, F$, and $\varphi$, but with the same t. By [12, Lemma 5], we have $E_{\lambda, N}(z, \omega)^{\sigma}=E_{\lambda, N}\left(z, \omega^{\sigma}\right)$ if $\lambda \neq 2$ or $\omega$ is nontrivial. This is so even when $\lambda=2$ and $\omega$ is trivial, if the action of $\sigma$ is 
defined by (3.12), (In fact, it is $\boldsymbol{Q}$-rational.) Therefore we see from (3) of Lemma 7 that $h^{\sigma}$ is exactly the form which plays the role of $h$ for $f^{\sigma}, F^{\sigma}$, and $\varphi^{\sigma}$. Thus we have

$$
R^{\prime} a\left(t, f^{\sigma}\right) A\left(m, F^{\sigma}, \varphi^{\sigma}\right)=\left[\varphi_{t}, \chi^{\sigma}, \varphi^{\sigma}\right]^{-1} P_{N}\left(\lambda, \omega^{\sigma}\right) \pi i \tau\left(\chi^{\sigma}\right)\left\langle h^{\sigma \rho}, f^{\sigma}\right\rangle .
$$

Define $I(f, g)$ by $(1.13)$; divide (4.8) by $u_{-}(F)$ and apply $\sigma$. Then we find, in view of $(1.12 \mathrm{~b})$ and $(3.6 \mathrm{a}, \mathrm{b})$, that

$$
\left[I\left(h^{\rho}, f\right) / u_{-}(F)\right]^{\sigma}=I\left(h^{\sigma \rho}, f^{\sigma}\right) / u_{-}\left(F^{\sigma}\right) .
$$

We have obtained $h$ for each fixed $t$ satisfying (4.5); now write $h_{t}$ for $h^{\rho}$. Observe that every positive integer can be written as $t n^{2}$ with $t$ satisfying (4.5) and a positive integer $n$ prime to $r$. Therefore (4.6) and (4.9) show that $f=0$ if $\left\langle h_{t}, f\right\rangle=0$ for all $t$ satisfying (4.5). It follows that the $h_{t} \operatorname{span} \mathcal{S}(k / 2, M, \chi, F)$.

Theorem 1 for $k \geqq 5$ now follows easily from this result. Changing the notation in order to avoid confusion, let $p \in \mathcal{S}(k / 2, M, \chi, F)$ and $q \in \mathcal{S}\left(k / 2, M^{\prime}\right)$. Then we have $p=\sum_{s \in V} b_{s} h_{s}$ and $q=r+\sum_{t \in V} c_{t} h_{t}$ with $b_{s}$ and $c_{t}$ in $C, r$ in $\mathscr{I}(k / 2, M, \chi, F)$ of Lemma 7 , and with a finite set $V$ of positive integers satisfying (4.5). Then

$$
I(p, q)=\sum_{s, t \in V} \bar{b}_{s} c_{t} I\left(h_{s}, h_{t}\right)
$$

By (4.10) and (3) of Lemma 7, we have

$$
\left[I(p, q) / u_{-}(F)\right]^{\sigma}=I\left(p^{\rho \sigma \rho}, q^{\sigma}\right) / u_{-}\left(F^{\sigma}\right)
$$

for every $\sigma \in \operatorname{Aut}(\boldsymbol{C})$. Now $K(F)$ is either totally real or is a $C M$-field. Therefore $K(F, \chi)=K\left(F^{\rho}, \chi^{\rho}\right) \subset K\left(p^{\rho}\right)$ by Lemma 8 , and hence (4.11) shows that $I(p, q) / u_{-}(F)$ belongs to $K\left(p^{\rho}, q\right)$. If $p$ is $K(F, \chi)$-rational, we have $K(p)=$ $K\left(p^{\rho}\right)=K(F, \chi)$ and $\rho \sigma \rho=\sigma$ on $K(p)$. This proves Theorem 1 when $k>3$.

Next let us assume $k=3$. In this case we have $m=1$ and $D(1, F, \varphi)$ may be 0 , and therefore it is necessary to modify the above reasoning. We first choose a character $\varphi_{1}$ under conditions $(4.4 \mathrm{a}, \mathrm{b})$. If $D\left(1, F, \varphi_{1}\right) \neq 0$, then the whole preceding argument is valid. (Since $\lambda=1, g \cdot E_{\lambda, N}(z, \omega)$ is always a cusp form.) Suppose $D\left(1, F, \varphi_{1}\right)=0$, and put

$$
F(z)=\sum_{n=1}^{\infty} c(n) e(n z), \quad F^{*}(z)=\sum_{n=1}^{\infty} \varphi_{1}(n) c(n) e(n z) .
$$

Applying [13, Theorem 2] to $F^{*}$, we find a character $\xi$ whose conductor is a prime not dividing $M t r^{2}$, and such that $\xi(-1)=1, D\left(1, F^{*}, \xi\right) \neq 0$. Put $\varphi=\xi \varphi_{1}$. Then

$$
D(1, F, \varphi)=D\left(1, F^{*}, \xi\right) \neq 0 .
$$

Therefore the above reasoning for $k>3$ is valid also in the case $k=3$ with this 
choice of $\varphi$. This completes the proof of Theorem 1.

REMARK. In the case $k>3$, we have employed a character $\varphi$ such that $\varphi^{2}=1$. Therefore the forms $h_{t}$ in the above proof are $K(\chi, F)$-rational.

\section{Proof of Theorems 2 and 3.}

We now go back to (4.1) and (4.2) with $k>3$. A direct calculation shows that

$$
E_{\lambda, N}(z,-r, \omega)=\frac{\Gamma(\lambda-2 r)}{\Gamma(\lambda-r)}(-4 \pi y)^{r} \delta_{\lambda-2 r}^{r} E_{\lambda-2 r, N}(z, \omega) \quad(0 \leqq 2 r<\lambda, r \in \boldsymbol{Z}) .
$$

Let $n$ be an odd integer such that

$$
(k+l-4) / 2<n \leqq k-2,
$$

and let $r=(k-n-2) / 2$. Then $0 \leqq 2 r<\lambda$ and $r \in Z$. Substituting $n / 2$ for $s$ in (4.1) and (4.2), we find that

$$
D(n / 2, f, g)=J \pi^{(k-1) / 2}\left\langle f^{\rho}, g \delta_{\lambda-2 r}^{r} E_{\lambda-2 r, N}(z, \omega)\right\rangle,
$$

where $J$ is a rational number depending only on $k, l, n$, and $N$. Now assume that $f \in \mathcal{S}(k / 2, M, \chi, F)$. Let $h$ be the essential part of $g \delta_{\lambda-2 r}^{r} E_{\lambda-2 r, N}(z, \omega)$ in the sense of Lemma 9, Observing that $h \in \mathcal{S}\left(k / 2, N, \chi^{\rho}\right)$, decompose $h$ into the sum $h=p+q$ with $p$ in $\mathcal{S}\left(k / 2, M, \chi^{\rho}, F^{\rho}\right)$ and $q$ in $\mathscr{I}\left(k / 2, M, \chi^{\rho}, F^{\rho}\right)$ of Lemma 7. We can now state a preliminary version to Theorem 2 as

THEOREM 4. Let $f \in \mathcal{S}(k / 2, M, \chi, F)$ and $g \in \mathscr{M}\left(l / 2, M^{\prime}\right)$ with odd integers $k$ and $l$ such that $0<l<k$ and $k \geqq 5$. For every positive integer $n$ satisfying (5.2), put

$$
B^{\prime}(n, f, g)=\pi^{(3-k) / 2} i \tau(\chi) D(n / 2, f, g) / u_{-}(F) .
$$

Then $B^{\prime}(n, f, g)^{\sigma}=B^{\prime}\left(n, f^{\sigma}, g^{\sigma}\right)$ for every $\sigma \in \operatorname{Aut}(C)$.

To prove this, we may assume that $g \in \mathscr{M}\left(l / 2, M^{\prime}, \phi\right)$ with a character $\phi$ modulo $M^{\prime}$. Then (5.3) shows that

$$
B^{\prime}(n, f, g)=J \cdot I\left(p^{\rho}, f\right) / u_{-}(F) .
$$

If we change $f$ and $g$ for $f^{\sigma}$ and $g^{\sigma}$, then $p$ is changed into $p^{\sigma}$. This follows from Lemma 12 and (3) of Lemma 7, Thus

$$
B^{\prime}\left(n, f^{\sigma}, g^{\sigma}\right)=J \cdot I\left(p^{\sigma \rho}, f^{\sigma}\right) / u_{-}\left(F^{\sigma}\right) .
$$

Hence our assertion follows immediately from Theorem 1.

The assertion of Theorem 2 for $n$ satisfying (5.2) is merely a restatement of this result. In fact, put $\varphi(d)=\left(\frac{-1}{d}\right)^{\lambda}$ and observe that $i^{\lambda} \tau(\varphi)$ is a rational 
number, and that $0<n+2-(k+l) / 2 \equiv \lambda(\bmod 2)$. Then

$$
B(n, f, g)=J^{\prime}[\chi, \phi, \varphi]^{-1} P_{N}(n+2-(k+l) / 2, \chi \psi \varphi) I\left(p^{\rho}, f\right) / u_{-}(F)
$$

with a rational number $J^{\prime}$ depending only on $k, l, n$, and $N$, and hence we obtain the desired conclusion from Theorem 1 and $(3.6 \mathrm{a}, \mathrm{b})$.

Now let $n^{\prime}$ be an odd integer such that $l \leqq n^{\prime}<(k+l) / 2$. The transformation $s \mapsto(1 / 2)(k+l)-1-s$ sends $n^{\prime} / 2$ to $n / 2$ with an odd integer $n$ satisfying (5.2). Therefore our result for $B\left(n^{\prime}, f, g\right)$ can be obtained by using the functional equation of $E_{\lambda, N}(z, s, \omega)$. The computation is exactly the same as what was done on [13, pp. 217-218]; we only have to take $k / 2, l / 2$, and $\chi \psi \varphi$ in place of $k, l$, and $\chi \psi$ there.

Finally, to prove Theorem 3 , we take $g$ from $\mathscr{M}\left(m, M^{\prime}, \phi\right)$ with an integer $m<k / 2$, and still $f$ in $\mathcal{S}(k / 2, M, \chi, F)$. In this case, a computation similar to that of $\S 4$ shows that

$$
(4 \pi)^{-s} \Gamma(s) D(s, f, g)=\int_{\Phi} \bar{f}_{\rho} g G_{\kappa, N}(z, 2 s+2-k, \omega) y^{k / 2} d \mu(z)
$$

where

$$
\begin{aligned}
& G_{\kappa, N}(z, s, \omega)=y^{s / 2} \sum_{\gamma \in W} \omega\left(d_{\gamma}\right) j(\gamma, z)^{-\kappa}|j(\gamma, z)|^{-2 s}, \\
& \kappa=k-2 m, \quad \omega(d)=\left(\frac{-1}{d}\right)^{m} \chi(d) \psi(d),
\end{aligned}
$$

and $W$ is a complete set of representatives of $\left[\{ \pm 1\} \Gamma_{1, \infty}\right] \backslash \Gamma_{0}(N)$. Now $G_{\kappa, N}(z, s, \omega)$ coincides with $E(z, s,-\kappa, \omega)$ of [10]; moreover, [10, Proposition 3] shows that

$$
(s+\alpha-1) \Gamma\left(\frac{s+\kappa}{2}\right) \Gamma\left(\frac{s+\alpha+\varepsilon}{2}\right) L_{N}\left(2 s+2 \alpha, \omega^{2}\right) E(z, s,-\kappa, \omega)
$$

is entire, where $\alpha=(\kappa-1) / 2$, and $\varepsilon$ is 0 or 1 according as $\alpha$ is even or odd; the factor $s+\alpha-1$ is necessary only when $\omega^{2}$ is trivial and $\kappa \equiv 1(\bmod 4)$. Therefore the first assertion of Theorem 3 follows from [10, Proposition 4] in exactly the same fashion as in $[10, \S 5]$.

Now put

$$
\begin{aligned}
& T_{1}=\left\{\begin{array}{l}
\{r \in 2 \boldsymbol{Z} \mid 0 \leqq r<(\kappa-3) / 2\} \text { if } \omega^{2} \text { is trivial, } \\
\{r \in 2 \boldsymbol{Z} \mid 0 \leqq r \leqq(\kappa-3) / 2\} \text { otherwise, }
\end{array}\right. \\
& T_{2}=\{r \in \boldsymbol{Z} \mid(\kappa-1) / 2 \leqq r<\kappa, r \equiv 1(\bmod 2)\}, \\
& H(z, r, \omega)=C_{r}\left\{L_{N}\left(2 s-1+\kappa, \omega^{2}\right) G_{\kappa, N}(z, s, \omega)\right\}_{s=-r},
\end{aligned}
$$


In [17], Sturm showed that $H(z, r, \omega)$ belongs to $\mathcal{A}_{\kappa}$, and moreover that $H(z, r, \omega)^{\sigma}=H\left(z, r, \omega^{\sigma}\right)$. These results can also be obtained by examining the Fourier expansion of the function $E^{*}(z, s,-\kappa, \omega)$ of [10] at $s=-r$ and applying Lemma 2 to it. This is similar to but somewhat more complicated than the analysis of $G^{*}(z, \omega)$ in the proof of Lemma 10. Anyhow, the arithmetic part of Theorem 3 can now be proved in the same fashion as for Theorem 2 by virtue of these properties of $H(z, r, \omega)$.

ADDENDUM. Some results of Reference [14] are quoted at several points in the present paper. We inform the reader that more than 20 misprints are in that article, though they affect neither the main theorems nor the results employed here. A full list of corrections has been sent to the Duke Mathematical Journal for publication.

\section{References}

[1] Y. Flicker, Automorphic forms on covering groups of $G L(2)$, preprint, 1979.

[2] S. Gelbart and I. Piatetski-Shapiro, On Shimura's correspondence for modular forms of half-integral weight, Proc. Int. Coll., Bombay, 1979.

[3] T. Miyake, On automorphic forms on $G L_{2}$ and Hecke operators, Ann. of Math., 94 (1971), 174-189.

[4] S. Niwa, Modular forms of half integral weight and the integral of certain thetafunctions, Nagoya Math. J., 56 (1975), 147-161.

[5] H. Petersson, Über die Entwicklungskoeffizienten der ganzen Modulformen und ihre Bedeutung für die Zahlentheorie, Abh. Math. Sem. Univ. Hamburg, 8 (1931), $215-242$.

[6] H. Petersson, Über die systematische Bedeutung der Eisensteinschen Reihen, Abh. Math. Sem. Univ. Hamburg, 16 (1949), 104-130.

[7] J.-P. Serre and H. Stark, Modular forms of weight 1/2, Modular functions of one variable VI, Lecture Notes in Math., 627 (1977), Springer, 27-67.

[8] G. Shimura, Introduction to the arithmetic theory of automorphic functions, Publ. Math. Soc. Japan, No. 11, Iwanami Shoten and Princeton Univ. Press, 1971.

[9] G. Shimura, On modular forms of half integral weight, Ann. of Math., 97 (1973), $440-481$.

[10] G. Shimura, On the holomorphy of certain Dirichlet series, Proc. London Math. Soc., 31 (1975) , 79-98.

[11] G. Shimura, Theta functions with complex multiplication, Duke Math. J., 43 (1976), 673-696.

[12] G. Shimura, The special values of the zeta functions associated with cusp forms, Comm. Pure Appl. Math., 29 (1976), 783-804.

[13] G. Shimura, On the periods of modular forms, Math. Ann., 229 (1977), 211-221.

[14] G. Shimura, The special values of the zeta functions associated with Hilbert modular forms, Duke Math. J., 45 (1978), 637-679.

[15] G. Shimura, On certain reciprocity-laws for theta functions and modular forms, Acta Math., 141 (1978), 35-71.

[16] T. Shintani, On construction of holomorphic cusp forms of half integral weight, Nagoya Math. J., 58 (1975), 83-126. 
[17] J. Sturm, Special values of zeta functions and Eisenstein series of half integral weight, Amer. J. Math., 102 (1980), 219-240.

[18] J. Sturm, Addendum to special values of zeta functions, Amer. J. Math., 102 (1980), 781-783.

\section{Goro SHIMURA}

Department of Mathematics

Princeton University

Princeton, New Jersey 08544

U.S. A. 